\title{
PREVENCIÓN DE INCENDIOS FORESTALES: ¿QUÉ ESTUDIAMOS? ¿COMO TRANSFERIMOS EL CONOCIMIENTO?
}

\section{Carmen Hernando Lara}

INIA. Centro de Investigación Forestal. Dpto. de Selvicultura y Gestión de los Sistemas Forestales. Laboratorio de Incendios Forestales. Carretera de A Coruña km 7,5. 28040-MADRID (España). Correo electrónico: lara@inia.es

\section{Resumen}

Se ha realizado una revisión de las principales bases de datos bibliográficas para registrar y cuantificar los estudios, tanto de carácter científico como técnico, que sobre incendios forestales, se vienen realizando en nuestro país, en los últimos años. Como principales fuentes de información se han manejado los trabajos publicados en: Revistas científicas especializadas (SCI); III, IV y V Congreso Forestal Espanol; $4^{\text {th }}, 5^{\text {th }}$ y $6^{\text {th }}$ International Conference on Forest Fire Research; $4^{\text {th }}$ y $5^{\text {th }}$ International Wildfire Conference y Revista Montes. Con esta revisión se pretende ofrecer un panorama sobre los esfuerzos que se están llevando a cabo en las tres grandes temáticas de los incendios: prevención, extinción y efectos/restauración, con especial énfasis en la prevención. Este ejercicio debería contribuir a la identificación de las futuras líneas prioritarias de investigación y de las vías más eficaces de transferencia del conocimiento; puntos cruciales para enfrentarnos al reto que supone la prevención de incendios forestales.

Palabras clave: Prevención, Convivir con el fuego, Gestión forestal sostenible, Incendios forestales

\section{INTRODUCCIÓN}

Los incendios forestales constituyen uno de los mayores problemas y amenazas a los que se enfrentan nuestros montes. A la alarma que supone una situación de emergencia, se unen los daños y perjuicios directos de bienes y aprovechamientos, y lo que es más importante, a largo plazo, las fuertes repercusiones sociales, económicas y ecológicas para los ecosistemas afectados. Diferentes instituciones en todos los ámbitos territoriales (local, regional, nacional y europea), gestores, tanto públicos como privados, e investigadores, destacan frecuentemente la problemática de los incendios y la necesidad de establecer protocolos de actuación, tanto antes (prevención), durante (emergencia) como después (restauración) de la perturbación, para de esta manera minimizar en lo posible los daños y consecuencias producidos.

Lo cierto es que en nuestro País, tras muchos años de desarrollo de importantes dispositivos de defensa contra incendios, se están desarrollando regímenes de fuego cada vez más severos, fundamentalmente como consecuencia del cambio climático y del uso actual del suelo, circunstancia que se reproduce en otros países mediterráneos (MOREIRA et al., 2011; PAUSAS \& FERNÁNDEZ-MuÑOZ, 2012; FERNANDES, 2013). Varios autores (PIÑOL et al., 2077; FERNANDES, 2008) han señalado que esta tendencia en los incendios, cada vez de mayor envergadura y más dañinos, es el resultado del desequilibrio de las políticas vigentes que van encaminadas a la 
supresión y no atienden lo suficientemente los aspectos preventivos.

Ante esta situación, se preconiza un cambio de paradigma y, de esta manera, las actuales directrices europeas apoyan el concepto de "Convivir con el fuego" (EFI, 2010), esto es, el desarrollo de estrategias a largo plazo dirigidas a actuar sobre las causas estructurales de los incendios e integrando la defensa contra incendios forestales en las estrategias de gestión forestal sostenible. Pero, este concepto de gestión integrada de incendios debe venir necesariamente secundado por una base científica y técnica sólida que fundamente el establecimiento de programas de actuaciones preventivas, priorizados y estables en el tiempo y ejecutados con criterios de eficiencia. Además, como señala BYROT (2009), la misión tradicional de la ciencia ha sido y es aportar conocimiento para apoyar la innovación, pero hoy en día también pasa por brindar experiencia a los procesos de elaboración de políticas.

Se ha destacado, frecuentemente, que las fuertes inversiones en la defensa contra los incendios forestales no han venido lo suficientemente acompañadas de inversiones en programas de investigación. No obstante, tanto la Comisión Europea, como el Plan Nacional y distintas instituciones nacionales y de las Comunidades Autónomas han financiado proyectos de investigación relevantes en materia de incendios que han generado importante información científica y técnica.

En el marco del proyecto FIRESMART, "Forest and land management options to prevent unwanted forest fires", financiado por el $7^{\circ}$ Programa Marco de la Unión Europea (20102012) y ejecutado por GMV, INIA, JRC, EIMFOR, FORESTIS, IRSTEA, Ambiente Italia y CEPF, se diseñó el cuestionario "FireSmart" sobre prevención de incendios forestales. El objetivo de dicho cuestionario era conocer la opinión de gestores públicos y privados e investigadores sobre la eficiencia y las consecuencias actuales de las prácticas de gestión en la prevención de incendios forestales en Europa, que permitieran identificar los obstáculos de tipo legal, socio-económico, técnico, etc. que están dificultando una prevención efectiva. El cuestionario constaba de 96 preguntas organizadas en bloques generales y temáticos. El análisis de las respuestas del colectivo español (286) fue publicado en un artículo de divulgación en la Revista Montes (HERNANDO et al., 2012). Cabe señalar que la pregunta: ¿En qué medida cree usted que la carencia de conocimientos científicos dificulta la prevención de los incendios forestales? obtuvo una puntuación media de 7 sobre 10 , lo que significa importantes carencias, no observándose diferencias entre las respuestas de los gestores y los investigadores. Los expertos opinaron, también, que la transferencia de conocimientos científicos a la gestión forestal no es lo suficientemente apropiada, ni lo suficientemente adaptada a las necesidades de la gestión.

\section{OBJETIVOS}

De acuerdo con lo anteriormente expuesto, en este trabajo se han explorado las principales bases de datos bibliográficas en materia de incendios forestales, con especial énfasis en el aspecto relativo a la prevención, para detectar y cuantificar las materias sobre las que las comunidades científica y técnica española vienen realizando más esfuerzos, en los últimos años. El ánimo de esta revisión es ofrecer un panorama que muestre las fortalezas y, lo que puede resultar más interesante, las principales carencias de información con el fin de identificar las futuras líneas prioritarias de investigación, desarrollo e innovación, así como la forma de llevar a cabo la transferencia del conocimiento a la gestión forestal.

\section{METODOLOGÍA}

Como principales fuentes de información se han manejado los trabajos publicados en:

- Revistas científicas especializadas (SCI), a través de la "web of knowledge".

- Actas del III, IV y V Congreso Forestal Español.

- Actas del $4^{\text {th }}, 5^{\text {th }}$ and $6^{\text {th }}$ International Conference on Forest Fire Research.

- $4^{\text {th }}$ and $5^{\text {th }}$ International Wildfire Conference

- Revista Montes.

Los trabajos registrados en cada una de las bases de datos se han clasificado, en un primer término, en las tres temáticas generales de incendios: prevención, supresión y efectos/restauración. En una segunda fase, los contempla- 
dos en el capítulo de prevención han sido desglosados por distintas actividades.

\section{RESULTADOS}

\section{Revistas científicas especializadas (SCI)}

En primer lugar, se ha realizado una búsqueda en la web of knowledge (Wok) de los artículos científicos publicados por autores españoles, durante el período 2009-2012. La media anual se sitúa en torno a los 40, mostrando una tendencia creciente durante dicho periodo (Figura 1), publicándose más de 50 artículos en 2012.

En esta búsqueda se han encontrado artículos españoles sobre incendios forestales en 78 revistas científicas pertenecientes a distintos ámbitos (forestal, ecología, medioambiente, historia, clima, geografía, botánica, teledetección...). Este dato pone de manifiesto el carácter multidisciplinar que tiene, en la actualidad, la ciencia del fuego. No obstante, solamente en 17 de las 78 revistas se han encontrado al menos tres publicaciones, durante el período de estudio. Las revistas con mayor número de artículos son: International Journal of Wildland Fire y Forest Ecology and Management con 22 y 17 artículos respectivamente (Figura 2).

En la Figura 3a se presenta la distribución de los artículos para cada una de las temáticas clásicas de los incendios forestales: prevención, comportamiento/extinción y efectos/restauración.

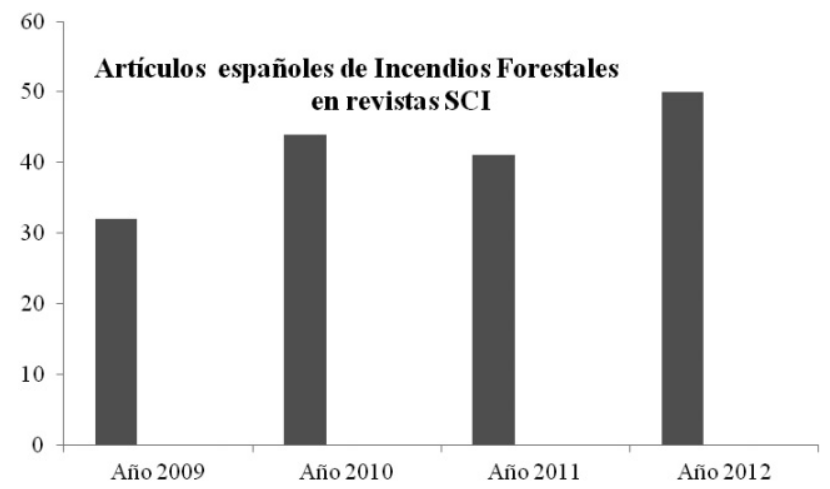

Figura 1. Evolución del número de artículos relativos a incendios forestales publicados por autores españoles en revistas cientificas SCI (2009-2012)

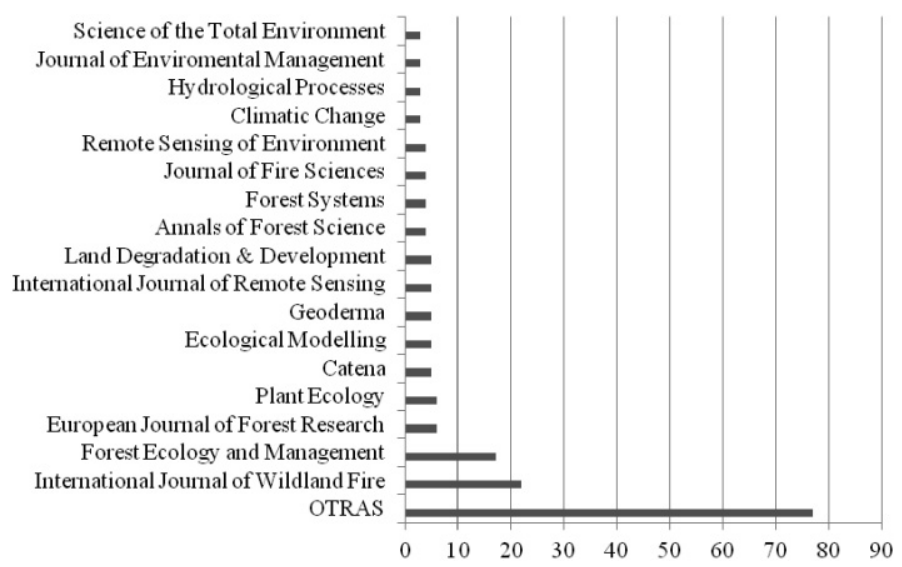

Figura 2. Revistas científicas (SCI) con al menos tres artículos publicados por autores españoles (2009-2012) 
Queda patente que es el capitulo de los efectos del fuego y la restauración de las zonas afectadas por los incendios el que resulta más productivo, desde un punto de vista científico, con un $66 \%$ de los artículos publicados concernientes a los incendios forestales, durante el período analizado. En el otro extremo se encuentran los artículos relacionados con el comportamiento del fuego y la extinción de incendios que representan solamente un $7 \%$ del total.

Por su parte, la Figura 3b muestra la distribución de los artículos encontrados en el capítulo de prevención agrupados en diferentes actividades: planificación, índices, gestión del combustible y detección de incendios. Como es obvio, en ambos casos, la asignación a una temática concreta resulta en ocasiones un poco artificiosa, teniendo en cuenta las estrechas interrelaciones existentes. En esta temática los artículos vínculados a la determinación de índices: riesgo, meteorologico, peligro, severidad, a través de diferentes metodologías, son los que tienen con diferencia mayor representación, con un $56 \%$ del total. Destacar que los trabajos relacionados con la gestión del combustible, aspecto que resulta clave en la modificación del régimen de incendios (FERNANDES, 2013), solamente suponen un $15 \%$ del total.

\section{III, IV y V Congreso Forestal Español}

La Figura 4 presenta la evolución del número total de comunicaciones y el número de comunicaciones sobre fuegos forestales recogidas en las Actas del III, IV y V Congreso Forestal. Destacar que a partir del IV Congreso (Zaragoza, 2005), en el que se instauró la Mesa Temática de Incendios Forestales, se produce un aumento importante del número de comunicaciones sobre incendios que representa aproximadamente un $10 \%$ del total de las comunicaciones, frente a un $4 \%$ registrado en las ediciones anteriores.

En la Figura 5 se muestra la distribución de las comunicaciones relativas a incendios forestales por temas: prevención, comportamiento/extinción y efectos/restauración para los tres congresos considerados en este estudio. Como se puede observar, no existen diferencias sustanciales entre congresos. El capítulo de comportamiento del fuego y extinción resulta en todas las ediciones el menos representado, si bien ha sufrido un incremento del $8 \%$ en la III edición al $17 \%$ en la V. Cabe destacar que los trabajos enmarcados en el grupo de efectos/restauración son predominantemente de carácter científico, mientras que las otras dos temáticas presentan muchas comunicaciones de carácter técnico y tecnológico.

a) Artículos españoles publica dos en revistas SCI (2009-2012)

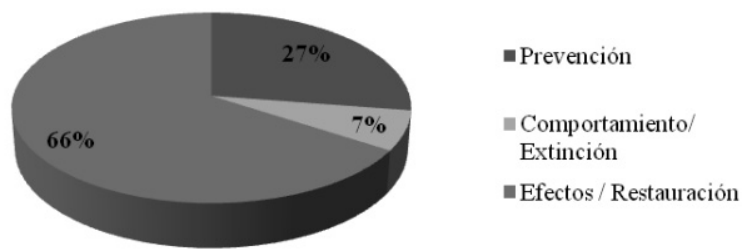

b) Artículos españoles publica dos en revistas SCI (2009-2012)

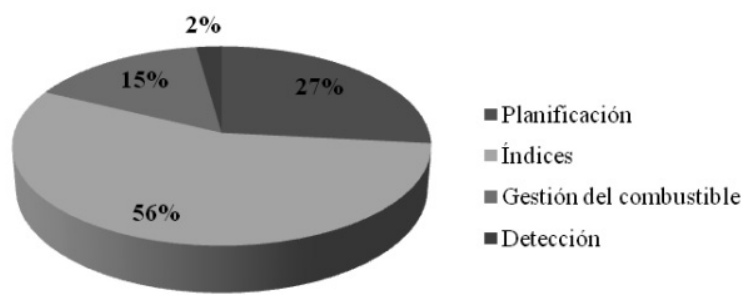

Figura 3. Distribución de artículos de autores españoles publicados en revistas SCI (2009-2012 a) por temáticas de incendios forestales y $b$ ) por temáticas relacionadas con la prevención 


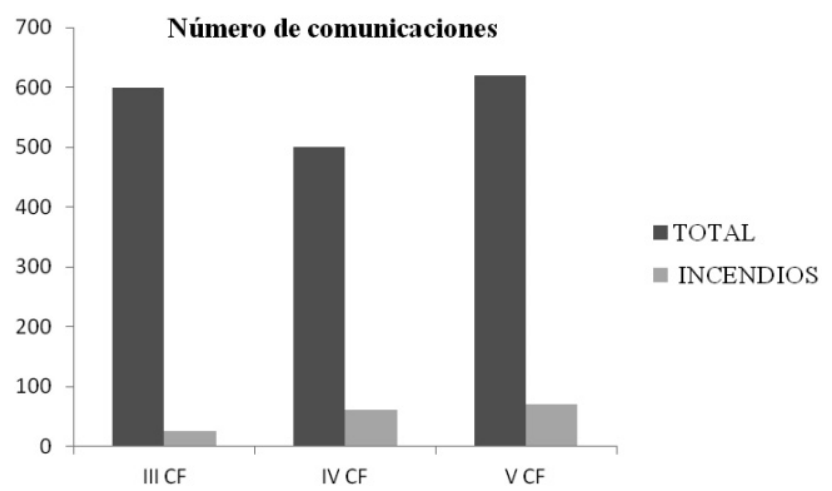

Figura 4. Número de comunicaciones totales y comunicaciones sobre incendios forestales (mesa de incendios y otras mesas) en el III, IV y V Congreso Forestal

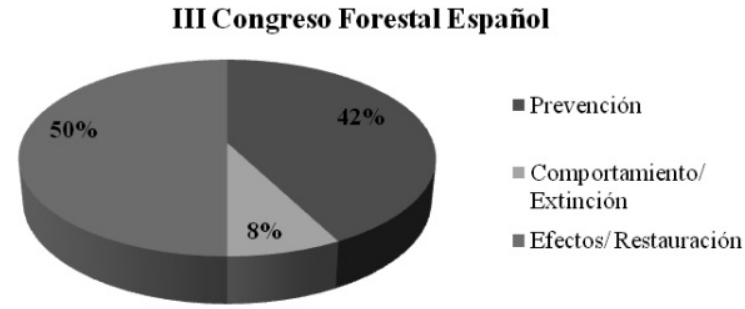

IV Congreso Forestal Español

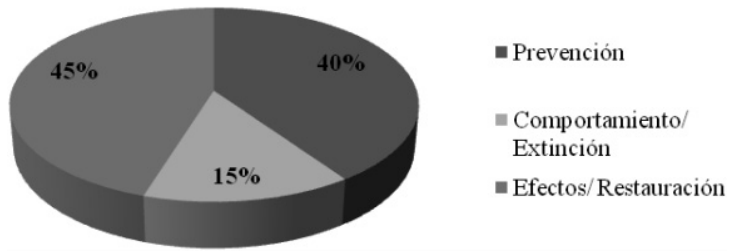

V Congreso Forestal Español

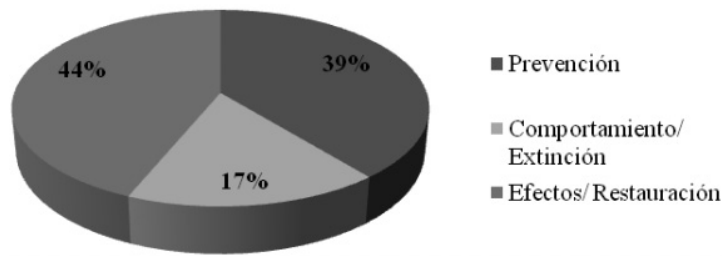

Figura 5. Distribución de comunicaciones por temáticas de incendios forestales, en el III, IV y V Congreso Forestal Español

En cuanto al ámbito de la prevención existen registros de trabajos en relación con: planificación, índices, gestión del combustible y detección de incendios. La Figura 6 muestra, el porcentaje de comunicaciones concerniente a cada una de las mencionadas materias preventivas, para cada uno de los tres congresos revisados. Destacar el aumento significativo del epígrafe planificación/gestión (40\%) en el V congreso en detrimento, sobre todo, de las relacionadas con la 
determinación de índices que en el III Congreso suponían el 55\% frente al 24\% del V (Figura 6).

\section{IV, V y VI International Conference on Forest Fire Research}

Este congreso internacional, altamente reconocido en materia de investigación sobre incendios forestales a nivel mundial, se lleva celebrando en Portugal desde 1990, cada cuatro años, con una participación importante de investigadores y técnicos españoles. Concretamente, se presentaron 51 comunicaciones españolas de un total de 193 en la edición IV (año 2002), 54 de 265 en la edición V (2006) y 28 de 241 en la VI (2010).

Cada una de las ediciones estuvo organizada en distintas Mesas Temáticas, con la distribución de comunicaciones que se muestra en la Figura 7. Como se puede observar la distribución de comu- nicaciones totales, autores españoles y no españoles, por temas de incendios ha ido cambiando, si bien, en parte es achacable a la variación en la temática de las mesas. Cabe destacar que los trabajos relacionados con el comportamiento del fuego tienen una presencia mucho mayor (entre el 23\% y el $30 \%$ ) que en las búsquedas presentadas anteriormente y que estaban referidas únicamente a autores españoles, lo que podría indicar un mayor interés por esta materia por parte de la comunidad científica internacional.

La Figura 8 muestra, asimismo, la distribución de comunicaciones por mesas temáticas, pero referida, en este caso, a las presentadas por autores españoles. Como se puede apreciar comparando las figuras 7 y 8 , en la IV edición la distribución es muy similar para las comunicaciones españolas y las totales. En la V edición la mayor

\section{Congreso Forestal Español PREVENCIÓN}

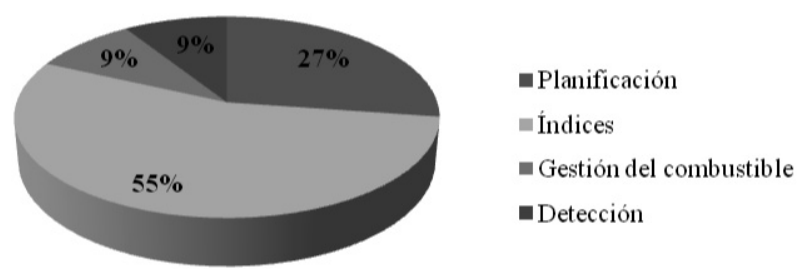

IV Congreso Forestal Español PREVENCIÓN

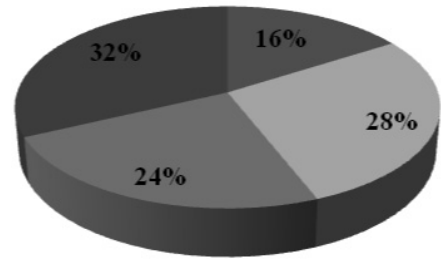

- Planificación

=Índices

- Gestión del combustible

Detección

\section{Congreso Forestal Español PREVENCIÓN}

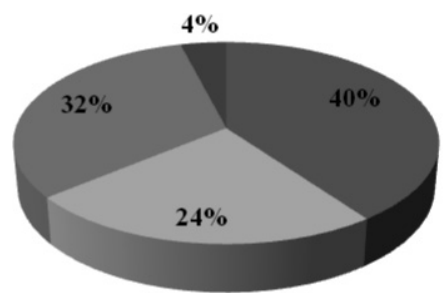

-Planificación

=Índices

- Gestión del combustible

Detección

Figura 6. Distribución de comunicaciones por temáticas relativas a la prevención de incendios, en el III, IV y V Congreso Forestal Español 


\section{Conference on Forest Fire Research (2002)}

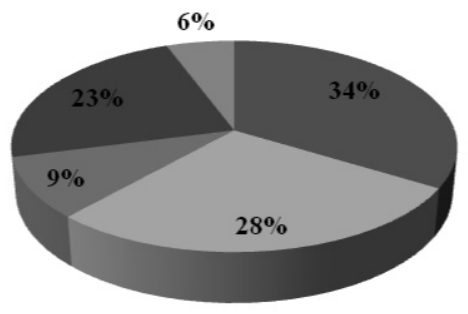

- Prevencióny gestión

- Comportamiento del fuego

Extincióny

seguridad

Efectos del fuego

Aspectos humanos $\mathrm{e}$ institucionales

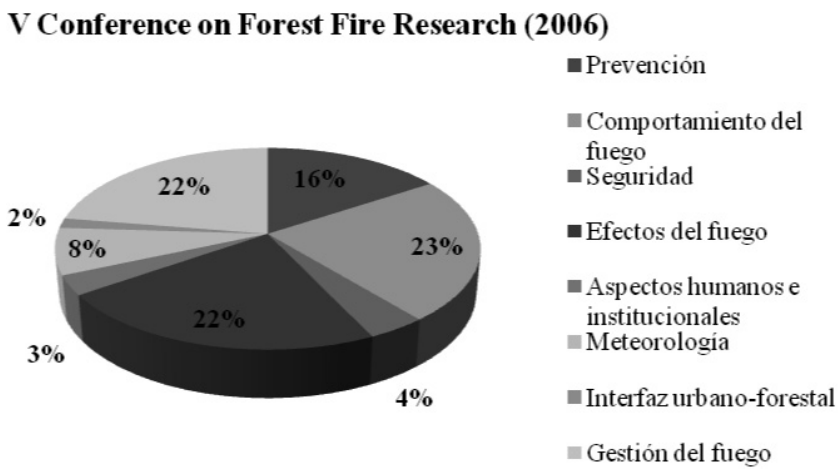

VI Conference on Forest Fire Research (2010)

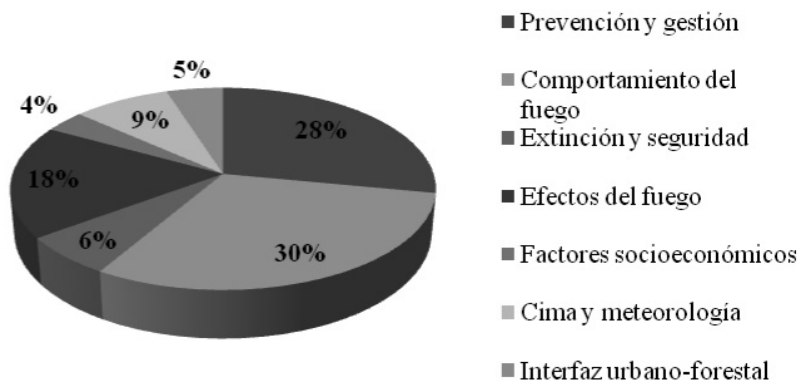

Figura 7. Distribución por sesiones temáticas del total de las comunicaciones presentadas a IV, V y VI International Conference on Forest Fire Research

diferencia se observa en el epígrafe de efectos del fuego que resulta superior en el caso de comunicaciones españolas. En la VI edición se detectan diferencias importantes en lo que se refiere a las comunicaciones del capítulo de seguridad y extinción ( $21 \%$ totales frente al $6 \%$ españolas) y en el de comportamiento del fuego (30\% totales frente al $19 \%$ españolas). Cabe destacar que los trabajos de autores españoles correspondientes a estas dos últimas temáticas suelen estar enmarcados en proyectos financiados por distintos progra- mas de la Unión Europea y son presentados en colaboración con investigadores de otros países.

Con respecto a las comunicaciones españolas relativas a temas preventivos, contenidas en la Mesa Temática de prevención, pero también en otras, la distribución muestra que el aspecto más representado, en todas las ediciones, por encima del $60 \%$, es el que se refiere a índices (Figura 9), como ya se detectó en el caso de los Congresos Forestales Españoles y las publicaciones en revistas científicas. También destacar 

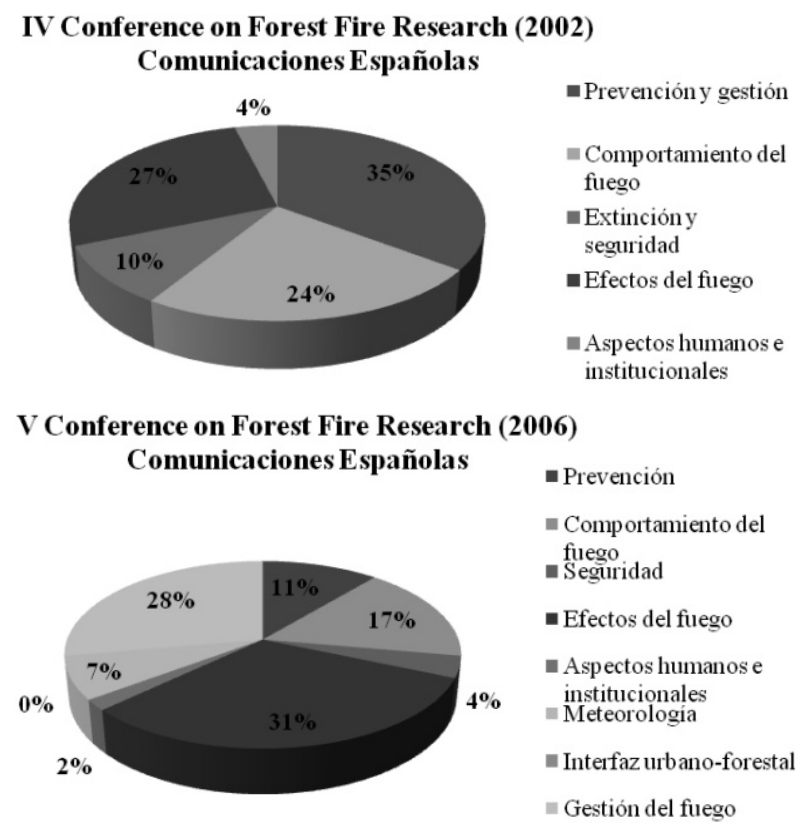

VI Conference on Forest Fire Research (2010) Comunicaciones Españolas

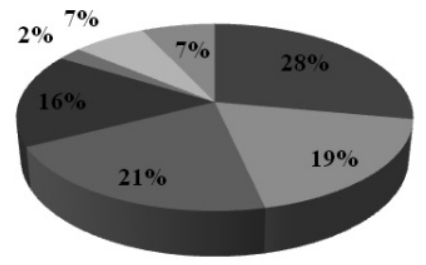

$$
\begin{aligned}
& \text { - Prevención y gestión } \\
& \text { = Comportamiento del } \\
& \text { fuego } \\
& \text { - Extinción y seguridad } \\
& \text { - Efectos del fuego } \\
& \text { - Factores } \\
& \text { socioeconómicos } \\
& \text { = Cima y meteorologia } \\
& \text { m Interfaz urbano-forestal }
\end{aligned}
$$

Figura 8. Distribución por sesiones temáticas del total de las comunicaciones españolas presentadas a IV, V y VI International Conference on Forest Fire Research

el incremento en el número de estudios relativos a la gestión del combustible.

\section{Conferencia Internacional sobre} Incendios Forestales: WILDFIRE, 2007

Cabe destacar que la cuarta Conferencia Internacional sobre Incendios Forestales, WILDFIRE 2007, se celebró en Sevilla lo que potenció, como no podía ser de otra manera, numerosos trabajos de carácter científico, tecnológico y técnico españoles. De un total de 424 comunicaciones, 226 fueron realizadas por autores de nuestro país.
La Figura 10a y 10b muestran la distribución de dichas comunicaciones por temáticas de incendios y por temáticas de materias relacionadas con la prevención respectivamente.

Por el contrario, la quinta conferencia WILDFIRE, 2011, tuvo lugar en Sudáfrica, bajo el lema "convivir con el fuego". La asistencia española fue prácticamente anecdótica.

\section{Revista Montes}

Durante el período 2009-2012, en la Revista Montes se han publicado 76 artículos de carácter 


\title{
IV Conference on Forest Fire Research (2002) \\ Comunicaciones Españolas en Prevención
}

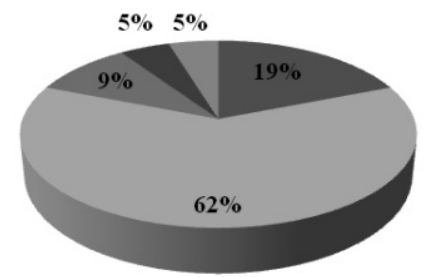

\author{
- Planificación \\ ándices \\ a Gestión del combustible \\ - Detección \\ a Concienciación
}

VConference on Forest Fire Research (2006)

Comunicaciones Españolas en Prevención
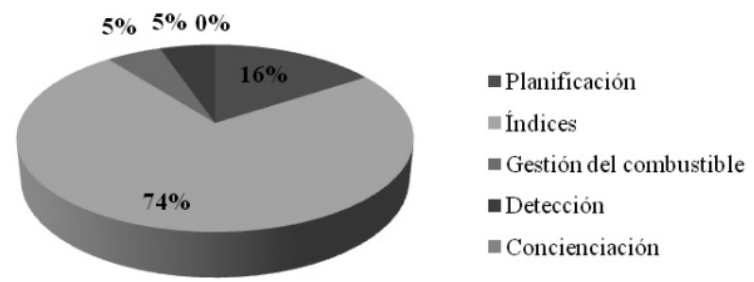

VI Conference on Forest Fire Research (2010)

Comunicaciones Españolas en Prevención

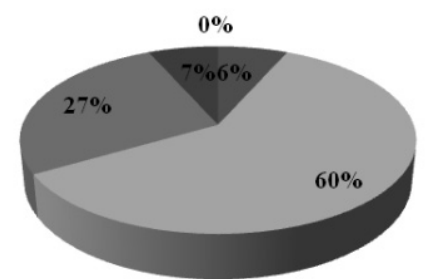

- Planificación
$\square$ Índices
$\square$ Gestión del combustible
$\square$ Detección
a Concienciación

Figura 9. Distribución de las comunicaciones españolas por temáticas relativas a la prevención de incendios presentadas a IV, Vy VI International Conference on Forest Fire Research

científico o divulgativo. Del total, diez artículos están centrados en los incendios forestales, lo que supone aproximadamente un $8 \%$. La mayoría de ellos se encuentran recogidos en un número especial de incendios que fue publicado en 2012.

\section{DISCUSIÓN Y CONCLUSIONES}

En primer lugar, cabe destacar que existe, en los últimos cuatro años, una tendencia creciente del número de publicaciones científicas españolas en revistas SCI lo que indicaría una mayor capacidad productiva de nuestra comunidad cientifíca. También queda patente un importante desequilibrio del número de artículos relativos a las distintas temáticas de los incendios: prevención, comportamiento/extinción y efectos/restauración, siendo el capitulo de los efectos del fuego y la restauración de las zonas afectadas por los incendios el que resulta más productivo, desde un punto de vista científico. Esta circunstancia ya fue detectada por VEGA et al. (1993) en una revisión realizada, a nivel mundial, para los trabajos recogidos en el Forestry Abstracts, entre los años 1952 y 1991. En el otro extremo se encuentran los artículos relacionados con el comportamiento del fuego y la extinción de incendios que representan solamente un $7 \%$ del total.

En relación a la prevención de incendios, la predicción del riesgo y del peligro de incendio, así como los sistemas de predicción meteorológi- 
a) IVWILDFIRE (Sevilla, 2007)

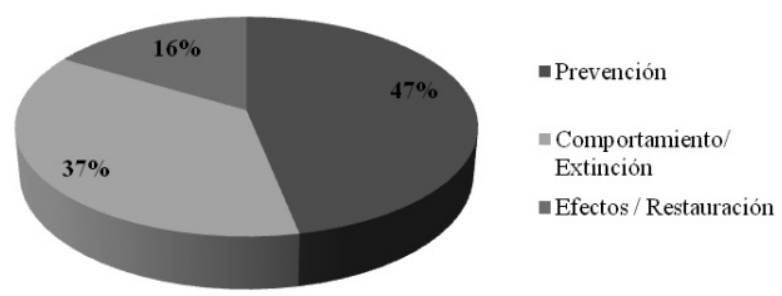

b) IVWILDFIRE (Sevilla, 2007) PREVENCIÓN
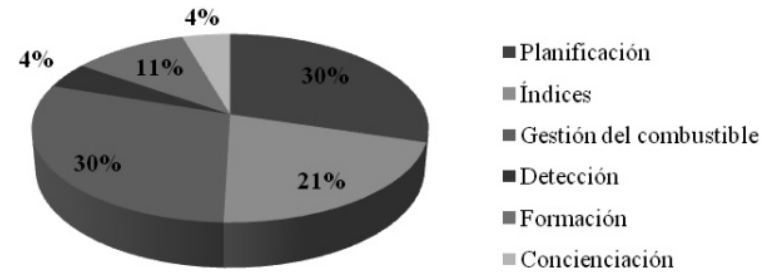

Figura 10. Distribución de las comunicaciones españolas a) por temáticas de incendios b) por aspectos preventivos

ca, es el aspecto al que se le dedica mayor esfuerzo tanto de investigación como de desarrollo.

Destacar, que los trabajos científicos relacionados con la gestión del combustible, aspecto clave en la modificación del régimen de incendios, solamente suponen un $12 \%$ del total. En concreto, los estudios experimentales del comportamiento del fuego y sus impactos en relación a los tratamientos de combustible son escasos, en nuestro país, pero también lo son a nivel mundial, por lo que sería una línea fundamental de investigación. En el contexto de cambio climático resulta aún más acuciante, la gestión selvícola, ya que los tratamientos preventivos pueden minimizar la probabilidad de incendios de copa. La complejidad de las actuaciones preventivas sobre la biomasa de un ecosistema queda de manifiesto en los modelos de simulación; existen múltiples modelos pero, a pesar de la experiencia recabada, todavía se necesita una sólida base de experimentación y validación de las simulaciones en campo (VEGA, 2011).

Teniendo en cuenta el altísimo porcentaje de fuegos no deseados relacionados con actividades y comportamientos humanos (VELEZ, 2009) las políticas a largo plazo deben focalizarse en la eliminación de causas. El análisis de las condiciones sociales, ecológicas y económicas que contribuyen al riesgo de los incendios deberían alimentar esas políticas, rompiendo con el modelo reactivo para adoptar otro de anticipación (BIROT \& RigOlOT, 2009). En consecuencia, resulta imprescindible fomentar la investigación en los aspectos sociales a los que hasta el momento no se les ha dedicado mucha atención.

Por otro lado, la economía relacionada con los incendios forestales debería dar respuestas respecto a los valores que estamos protegiendo y no sólo a las pérdidas derivadas de los mismos (MAVSAR, 2009). La falta de mecanismos de valoración económica de la inversión en prevención, es bastante patente, por lo que es fundamental analizar los aspectos coste/beneficio de la prevención. Para ello, resulta necesario implementar un sistema de información que recoja los costes económicos de las acciones de prevención de incendios, así como un sistema de indicadores que permita evaluar la eficiencia de dichas acciones, aspecto al que se ha dedicado pocos esfuerzos.

Finalmente, a pesar del incremento de episodios de incendios urbano-forestales, no existe una conciencia social ni una verdadera preocupación política sobre el problema (GALIANA, 2012). Si bien, existen regulaciones sobre la gestión del combustible en la interfaz urbano-forestal su aplicación es escasa (MoreIRA et al., 2011), debido en gran medida a las limitaciones en los recursos, la 
débil aplicación de la ley y la escasa concienciación de los residentes (GILL \& STEPHENS, 2009).

Como ha sido mencionado anteriormente, la misión de la ciencia hoy en día, también, pasa por brindar experiencia a los procesos de elaboración de políticas, por lo que resulta imprescindible crear una ciencia del fuego, participada por todos los agentes implicados, que considere las importantes carencias señaladas y que secunde el concepto de gestión integrada de incendios.

Lo expuesto, hasta ahora, se podría resumir en la necesidad de:

- Potenciar canales bidireccionales de comunicación eficaces entre gestores e investigadores para priorizar las líneas de investigación y asegurar la transferencia del conocimiento.

- Fomentar el análisis de las condiciones ecológicas, sociales, legales y económicas que contribuyen al riesgo.

- Introducir mecanismos para implementar la gestión sostenible de los sistemas forestales, integrando el conocimiento científico y técnico.

- Fomentar la investigación de los aspectos coste/beneficio de la prevención, así como de indicadores que permitan evaluar la eficiencia de dichas acciones.

- Incrementar los estudios experimentales del comportamiento del fuego y sus impactos en relación a los tratamientos de combustible.

- Fomentar la conexión de las comunidades involucradas en el sector forestal asegurando una transmisión del conocimiento eficaz.

- Fomentar programas de investigación que apoyen la elaboración de estrategias adecuadas para educar (informar y concienciar) a la sociedad en las cuestiones relacionadas con el sector forestal en general y la prevención de los incendios forestales en particular.

\section{BIBLIOGRAFÍA}

Birot, Y.; 2009. Convivir con los incendios forestales: lo que nos revela la ciencia. EFI Discussion Paper 15: 1-79.

Birot, Y. \& Rigolot, E.; 2009. La necesidad de estrategias que se anticipen al cambio climático entre otros. En: Convivir con los incendios forestales: lo que nos revela la ciencia. EFI Discussion Paper 15: 82-85.
EFI; 2010. A Mediterranean Forest Research Agenda-MFRA. European Forest Institute. Joensuu.

FERNANDES, P.M.; 2008. Forest fires in Galicia (Spain): The outcome of unbalanced fire management. Journal of Forest Economics 14: $155-157$.

FERNANDES, P.M.; 2013. Fire-smart management of forest landscapes in the Mediterranean basin under global change. Landscape Urban Plan. 110: 175-182.

GALIANA, L.; 2012. Las interfaces urbano-forestales: un nuevo territorio de riesgo en España. Boletín de la Asociación de Geógrafos Españoles 58: 205-226.

GiLl, A.M. \& STEPHENS, S.L.; 2009. Scientific and social challenges for the management of fire-prone wildland-urban interfaces. Environ Res. Letters 4: 034014.

Hernando, C; Planelles, R.; Ortega, M.; Garrido, S.; Madrigal, J.; GUIJARro, M.; Aragonés, J.I. \& Sebastián, A.; 2012. La opinión de los gestores e investigadores sobre la prevención de incendios forestales en España: resultados del cuestionario "FIRESMART". Montes 109: 33-38.

MAVSAR, R.; 2009. Aspectos económicos de los Incendios Forestales. En: Convivir con los incendios forestales: lo que nos revela la ciencia. EFI Discussion Paper 15: 66-67.

Moreira, F.; Viedma, O.; Arianoutsou, M.; Curt, T.; Koutsias, N.; Rigolot, E.; 2011. Landscape-wildfire interactions in southern Europe: Implications for landscape management. J. Environ. Manage. 92: 2389-2402.

PAusas, J.G. \& Fernández-Muñoz, S.; 2012. Fire regime changes in the Western Mediterranean Basin: from fuel-limited to drought-driven fire regime. Climate Change 110: 215-226.

Piñol, J.; Castellnou, M. \& Beven, K.J.; 2007. Conditioning uncertainty in ecological models: Assessing the impact of fire management strategies. Ecol. Model. 207: 34-44.

Vega, J.A., CUIÑAS, P.; FontúRBEl M.T. y GRAS, J.M.; 1993. El estado actual sobre fuegos forestales: Evaluación de lo realizado y prioridades futuras. En: F.J. Silva-Pando y G. Vega Alonso (eds.), Ponencias y Comunicaciones. 
Congreso Forestal Español Lourizán 1993, III: 263-268. Grapol S.A. Vigo.

VÉLEZ, R.; 2009. Los factores causantes: las fuerzas y cambios sociales y económicos. En: Convivir con los incendios forestales: lo que nos revela la ciencia. EFI Discussion Paper 15: 24-27.
Actas del III, IV y V Congreso Forestal Español. Sociedad Española de Ciencias Forestales. Actas WILDFIRE; 2007.

Proceedings IV, V y VI Conference on Forest Fire Research (Ed. D.X. Viegas) http://www.firesmart-project.eu http://wokinfo.com/ 